\title{
CARACTERIZAÇÃO DOS EPISÓDIOS DE QUEDA EM UMA UNIDADE DE CARDIOLOGIA: ESTUDO RETROSPECTIVO
}

\section{CHARACTERIZATION OF FALLS IN A CARDIOLOGY UNIT: A RETROSPECTIVE STUDY}

\section{CARACTERIZACIÓN DE EPISODIOS DE CAÍDAS EN UNA UNIDAD DE CARDIOGOLÍA: ESTUDIO RESTROSPECTIVO}

Andreia Lima de Sousa ${ }^{1}$, Ana Carolina Lici Monteiro², Maria Leticia Banwart Ribeiro Ambiel${ }^{3}$, Elaine Barros Ferreira ${ }^{4}$, Priscilla Roberta Silva Rocha ${ }^{5}$

\section{RESUMO}

Objetivo: Caracterizar os episódios de queda em pacientes internados em uma unidade cardiológica, quanto à ocorrência, fatores relacionados e risco. Método: Estudo descritivo, retrospectivo, por meio da análise de prontuários de pacientes internados em uma unidade de cardiologia que apresentaram episódio de queda entre janeiro de 2015 a dezembro 2016 . O risco médio para quedas foi avaliado, conforme a Escala de Morse. Resultados: No período de estudo houve 32 episódios de quedas. A queda foi mais frequente em idosos $(81,3 \%)$ e naqueles que faziam uso crônico de medicamentos, para controle e tratamento de comorbidades preexistentes. Dentre os fatores de risco, $34,4 \%$ apresentavam delirium, comprometimento neurológico e déficit de locomoção. 0 risco médio para quedas foi classificado como elevado ( $\geq 45), 25 \%$ das quedas resultaram em algum tipo de dano (leve ou moderado) e ocorreram em períodos matutinos. Conclusão: As contribuições fornecidas pelos registros de eventos adversos, deste estudo, facilitaram a identificação dos fatores de risco, demonstrando a necessidade de se propor intervenções de enfermagem preventivas, uma vez que assumir o evento e identificar suas causas são maneiras de praticar uma assistência de enfermagem segura ao paciente.

Descritores: Acidentes por Quedas; Fatores de Risco; Segurança do Paciente; Medidas de segurança; Cuidados de enfermagem; Cardiologia.

\begin{abstract}
Objective: To characterize the episodes of falls in patients hospitalized in a cardiology unit, regarding occurrence, related factors and risk. Method: This is a descriptive, retrospective study, analyzing medical records of patients admitted to a cardiology unit who experienced a fall episode between January 2015 and December 2016. The average risk for falls was assessed according to the Morse Scale. Results: During the study period, there were 32 episodes of falls. Falls were more frequent in the elderly people (81.3\%) and in those people who used chronic drugs to control and treat pre-existing comorbidities. Among the risk factors, $34.4 \%$ had delirium, neurological impairment and impaired mobility. The average risk for falls was classified as high (> 45 ), $25 \%$ of the falls resulted in some type of damage (mild or moderate) and occurred in the morning. Conclusion: The contributions provided by the records of adverse events in this study facilitated the identification of risk factors, demonstrating the need to propose preventive nursing interventions, since assuming the event and identifying its causes are ways to practice safe nursing care to the patient.
\end{abstract} Descriptors: Accidental Falls; Risk Factors; Patient Safety; Security Measures; Nursing Care; Cardiology.

\section{RESUMEN}

Objetivo: Caracterizar los episodios de caídas en pacientes hospitalizados en una unidad de cardiología, en cuanto a ocurrencias, factores relacionados y riesgo. Método: Estudio descriptivo, retrospectivo, mediante el análisis de las historias clínicas de los pacientes ingresados en una unidad de cardiología que experimentaron un episodio de caída entre enero de 2015 y diciembre de 2016. El riesgo promedio de caídas se evaluó según la escala de Morse. Resultados: Durante el período de estudio hubo 32 episodios de caídas. La caída fue más frecuente en los ancianos $(81,3 \%)$ y en los que usaban fármacos crónicos para controlar y tratar las comorbilidades preexistentes. Entre los factores de riesgo, el 34,4\% presentaba delirio, deterioro neurológico y movilidad reducida. El riesgo promedio de caídas se clasificó como alto (>45), el $25 \%$ de las caídas resultaron en algún tipo de daño (leve o moderado) y ocurrieron en la mañana. Conclusión: Los aportes que brindan los registros de eventos adversos en este estudio facilitaron la identificación de factores de riesgo, demostrando la necesidad de proponer intervenciones de enfermería preventivas, y a que asumir el evento e identificar sus causas son formas de practicar al paciente cuidados de enfermería seguros.

Descriptores: Accidentes por Caídas; Factores de Riesgo; Seguridad del Paciente; Medidas de Seguridad; Atención de Enfermería; Cardiología.

${ }^{1}$ Graduação em Enfermagem pela Universidade de Brasília. ${ }^{2}$ Graduação em Enfermagem pela Universidade Estadual do Norte do Paraná, especialista em enfermagem cardiovascular, modalidade residência, pelo IDPC - Instituto Dante Pazzanese de Cardiologia de São Paulo. ${ }^{3}$ Enfermeira, Especialista em enfermagem cardiovascular, Hospital do Coração do Brasil, Brasília, Distrito Federal, Brasil. ${ }^{4}$ Enfermeira, Doutora em Enfermagem pela Universidade de Brasília, DF, Brasil. Professora Adjunta, Departamento de Enfermagem, Universidade de Brasília. ${ }^{5}$ Enfermeira, Doutora em Ciências da Saúde pela Universidade de Brasília, DF, Brasil. Professora Adjunta do Curso de Enfermagem - Universidade de Brasília.

\section{Como citar este artigo:}

Sousa AL, Monteiro AC, Ambiel MLBR, et al. Frequência de quedas em pacientes internados em uma unidade de cardiologia: estudo retrospectivo. Revista de Enfermagem do Centro-Oeste Mineiro. 2020;10:e4059. [Access http://doi.org/10.19175/recom.v10i0.4059 ]; Available in: DOI: 


\section{INTRODUÇÃO}

Segundo a Organização Mundial de Saúde (OMS), ${ }^{(1)}$ o evento adverso consiste em um incidente decorrente da assistência que resulta em danos não intencionais ao paciente, e que não se relaciona à evolução natural da doença de base. Dados americanos reportam que $3,7 \%$ das internações, evoluem com presença de eventos adversos associados a danos, $6,5 \%$ desses eventos provocam disfunções permanentes e $13,6 \%$ envolvem a morte do paciente ${ }^{(2)}$. No Brasil, 7,6\% dos pacientes internados apresentam eventos adversos, sendo $66,7 \%$ desses episódios evitáveis ${ }^{(3)}$.

A Agência Nacional de Vigilância Sanitária $(A N V I S A)^{(4)}$, juntamente com o Ministério da Saúde $(M S)^{(5)}$, por meio da portaria 529 , de 1a abril de 2013, projetaram programas e estratégias de saúde voltadas para a segurança do paciente. Dentre os eixos estabelecidos, encontram-se: identificação correta do paciente, a melhor comunicação entre os profissionais de saúde, a melhoria na segurança na prescrição, no uso e na administração de medicamentos, a garantia de uma cirurgia segura, a higienização das mãos para a prevenção das infecções e a redução do risco de quedas e lesão por pressão(4-6).

A queda constitui um dos eventos adversos mais prevalentes no âmbito hospitalar, responsável por dois a cada cinco eventos indesejáveis ${ }^{(7-8)}$. A ANVISA reportou em um relatório sobre assistência hospitalar, que $38,2 \%$ dos eventos adversos correspondem às quedas ${ }^{(5)}$. A queda é conceituada como um deslocamento não intencional do corpo para um nível inferior à posição inicial, decorre de circunstâncias multifatoriais que comprometem a estabilidade e a manutenção do bem-estar do indivíduo ${ }^{(9)}$.

É um incidente complexo, não intencional, traumático e multifatorial que pode ser causado por diversos fatores, sejam eles intrínsecos ou extrínsecos ${ }^{(10-12)}$. Os fatores intrínsecos são aqueles relacionados com as alterações fisiológicas decorrentes do processo de envelhecimento, ao sexo, uso de medicamentos e condições patológicas. Dentre as condições patológicas, as mais frequentes são doenças cardiovasculares, uso de dispositivos, alteração na marcha, deficiência ocular, auditiva e cognitiva, mobilidade prejudicada e histórico anterior de quedas. Os fatores extrínsecos estão associados a questões ambientais, como inadequações arquitetônicas e de mobiliário, as quais a maioria dos idosos está exposta ${ }^{(12-13)}$.
A ocorrência de queda repercute de forma negativa, uma vez que pode associar-se a ocorrência de danos, por vezes permanentes e com gravidade variável, impactando no prolongamento da estadia hospitalar e em desfechos clínicos desfavoráveis ${ }^{(14)}$. A presença de fatores de risco para quedas é comum no ambiente hospitalar, variando conforme as características clínicas dos pacientes e do perfil epidemiológico específico de cada serviço, podendo atingir taxas entre 1,4 a 10,7 quedas/1.000 pacientes-dia ${ }^{(8,15)}$.

A utilização de instrumentos para a estratificação do risco de quedas, permite o estabelecimento da vulnerabilidade de cada indivíduo e favorece a elaboração de um plano de cuidados individual e assertivo. A Escala de Morse, Morse Fall Scale (MFS) $)^{(16-17)}$, é comumente utilizada como instrumento de estratificação de risco, categorizando os fatores que predispõem a queda e identificando o grau de risco do indivíduo ${ }^{(18-19)}$.

A presença de comorbidades cardiológicas está associada a vários fatores de risco para queda, sendo estes inerentes à própria doença ou relacionados aos métodos terapêuticos, em especial farmacológicos, uma vez que é comum o uso de medicamentos que, potencialmente, alteram a homeostase hemodinâmica, o débito cardíaco e a perfusão cerebral(20). Embora as escalas preditoras de risco avaliem fatores de risco para queda, a identificação de características clínicas, em uma população específica de pacientes que apresentaram o evento adverso queda, auxilia na identificação dos fatores de risco específicos, daquele grupo, além de direcionar as intervenções de enfermagem preventivas adequadas a esses pacientes.

Assim, objetivou-se, neste estudo, caracterizar os episódios de queda em pacientes internados em uma unidade cardiológica, quanto à ocorrência, fatores relacionados e risco.

\section{MÉTODO}

Trata-se de um estudo descritivo, retrospectivo, observacional, em uma unidade de internação cardiológica de um hospital terciário privado do Distrito Federal, para responder à seguinte pergunta de pesquisa: Quais são as características clínicas e fatores de risco apresentados pelos pacientes com episódio de queda na referida unidade?. O estudo foi conduzido de acordo com as diretrizes STROBE 
Statement ${ }^{(21),}$ a qual indica os pontos essenciais para a condução de um estudo observacional.

Foram analisados os episódios de queda, ocorridos em pacientes admitidos na unidade cardiológica, entre janeiro de 2015 a dezembro 2016, que apresentaram, pelo menos. um episódio de queda, durante a internação. Os episódios de queda eram, diariamente, monitorizados, pelo núcleo de segurança do paciente do hospital.

A coleta de dados se deu, no ano de 2017, por meio da análise do prontuário eletrônico. Para os pacientes que apresentaram o evento de interesse, foram coletados, dados clínicos (diagnóstico de internação, tempo de internação, medicações em uso), características demográficas (idade, sexo) e características relacionadas ao evento da queda (motivo, horário e local da queda, presença de comprometimento neurológico, presença de prejuízo de locomoção, pontuação de risco de queda, segundo Escala de Queda de Morse (MSF) ${ }^{(19)}$; e a ocorrência ou não de dano, com o intuito de descrever as características e fatores de risco mais prevalentes nos pacientes que apresentaram episódios de queda. As variáveis foram coletadas, utilizando-se instrumento estruturado elaborado pelos autores.

A MSF é constituída por seis itens com duas ou três possibilidades de resposta para cada um deles, correspondente a uma determinada pontuação. Os itens avaliados versam sobre: histórico de quedas, presença de diagnóstico secundário, grau de necessidade para auxílio na deambulação, uso de terapia endovenosa contínua ou dispositivo endovenoso salinizado/heparinizado, qualidade da marcha e estado mental. De acordo com a avaliação efetuada, a soma das pontuações obtidas, em cada um dos seis itens, resulta num score que indica o risco de queda. Os valores de referência para o risco de quedas são: $<24$ = risco baixo; 25 44 = risco moderado; $>45$ = risco elevado.

Os dados referentes à estratificação do risco de quedas, por meio da MSF, foram avaliados em três momentos: no dia que antecedeu a queda, no dia da queda e no dia seguinte a queda.

Os dados coletados foram transcritos para uma planilha eletrônica e exportados para Graphpad Prism versão 7.0. A caracterização da amostra foi feita, por meio de estatística descritiva. As variáveis contínuas foram reportadas como medida de tendência central e de dispersão (média, mediana e desvio padrão) e as variáveis categóricas por medida de frequência e porcentagem.

A pesquisa foi submetida e aprovada pelo Comitê de Ética em Pesquisa (CEP), sob protocolo na 58103816.8.0000.5667 respeitando a resolução 466 de 2012.

\section{RESULTADOS}

No período de estudo, houve 14.236 internações na unidade ( 6.894 no ano de 2015 e 7.252 em 2016) e 32 episódios de queda. A taxa de quedas na unidade foi de 2,7 quedas $/ 1000$ pacientes-dia em 2015 e de 1,5/1000 pacientesdia em 2016. Em 2015, ocorreram 24 episódios de quedas (75\% do total de eventos), a maioria idosos, com média de idade de 72,1 (DP 4,3), 26 $(81,3 \%)$ eram maiores de 60 anos. Em relação aos motivos admissionais, seis (19\%) desses pacientes foram admitidos, após procedimentos cirúrgicos/invasivos (revascularização miocárdica, angiografia e angioplastias) (Tabela 1).

Tabela 1 - Características clínicas e demográficas dos pacientes que apresentaram episódio de queda em uma unidade de internação cardiológica. Brasília, DF, Brasil, 2016.

\begin{tabular}{lc}
\hline \hline Características & Amostra $(\mathbf{n}=\mathbf{3 2})$ \\
\hline Sexo, $\mathrm{n}$ masculino (\%) & $16(50)$ \\
Idade em anos, média (DP)* & $72,1(14,3)$ \\
Faixa etária, $\mathrm{n}(\%)$ & $1(3,1)$ \\
$\quad<20$ anos & $0(0)$ \\
21 a 40 anos & $5(15,7)$ \\
41 a 60 anos & $26(81,3)$ \\
$\quad>6$ anos & \\
Diagnostico de internação, $\mathrm{n}(\%)$ & $26(81,3)$ \\
$\quad$ Clínico & $6(19,0)$ \\
$\quad$ Cirúrgico & \\
\hline \hline
\end{tabular}

Fonte: Dados internos do hospital referente aos episódios de quedas ocorridos entre 2015-2016.

*DP - desvio padrão

A agitação e o delirium, bem como o comprometimento neurológico e o déficit de locomoção foram identificados em 11 (34,4\%) pacientes, ao passo que $31(96,6 \%)$ dos pacientes utilizavam medicamentos de uso prolongado/crônico para controle e/ou 
tratamento das comorbidades preexistentes, em especial cardiológicas e metabólicas. O tempo médio de internação foi de 18,6 (DP 17,7) dias, não foi avaliado o incremento do tempo de internação como consequência do episódio de queda.
A pontuação média da MFS foi avaliada em três ocasiões: no dia do episódio de queda, no dia que antecedeu e sucedeu o evento (Tabela 2).

Tabela 2 - Estratificação do risco de queda e presença de dano em pacientes que apresentaram episódio de queda em uma unidade de internação cardiológica. Brasília, DF, Brasil, 2016.

\begin{tabular}{lc}
\hline \hline Características & Amostra $(\mathbf{n}=\mathbf{3 2})$ \\
\hline Escala de Morse (MFS) antes do evento, média (DP) & $47,4(22,4)$ \\
Escala de Morse (MFS) no dia do evento, média (DP) & $49,2(25,7)$ \\
Escala de Morse (MFS) após o evento, média (DP) & $48,8(25,4)$ \\
Ocorrência de dano, $\mathrm{n}(\%)$ & \\
$\quad$ Sem danos & $24(75)$ \\
$\quad$ Dano Leve & $5(15,6)$ \\
Dano moderado & $3(9,4)$ \\
Dano grave & $0(0)$ \\
\hline \hline
\end{tabular}

Fonte: Dados internos do hospital referente aos episódios de quedas ocorridos entre 2015-2016.

*DP - desvio padrão.

Em todos os momentos avaliados, o escore médio retratava um risco elevado para a ocorrência de quedas ( $\geq 45)$, entretanto, vale ressaltar que, no dia do evento, $15(46,9 \%)$ pacientes apresentavam risco elevado para queda (MFS $\geq 45$ ). Das quedas avaliadas, apenas 8 (25\%) resultaram em algum tipo de dano, destes prevaleceram os danos leves $5(15,6 \%)$, seguidos por danos moderados 3 (9,4\%) (Tabela 2 ).

Observou-se que $4(12,5 \%)$ dos pacientes já apresentavam histórico prévio de quedas. As Tabela 3 - Frequência das características relacionadas ao episódio de queda em pacientes uma unidade de internação cardiológica. Brasília, DF, Brasil, 2016.

\begin{tabular}{lc}
\hline \hline Características & Amostra $\mathbf{n}=\mathbf{3 2})$ \\
\hline Horário do episódio de queda, $\mathbf{n}(\%)$ & $10(31,2)$ \\
Manha & $7(21,9)$ \\
Tarde & $8(25)$ \\
Noite & $7(21,9)$ \\
Madrugada & \\
História prévia de queda, $\mathbf{n}(\%)$ & $4(12,5)$ \\
Sim & $28(87,5)$ \\
Não & \\
Local da queda, $\mathbf{n}(\%)$ & $11(34,6)$ \\
Cama & $12(37,5)$ \\
Banheiro & $1(3,1)$ \\
Poltrona & $1(3,1)$ \\
Balança & $1(3,1)$ \\
Corredor & $5(15,5)$ \\
Quarto & $1(3,1)$ \\
Enfermaria &
\end{tabular}

Fonte: Dados internos do hospital referentes aos episódios de quedas entre 2015-2016.

\section{DISCUSSÃO}

Estudos demonstram que síndromes geriátricas são bastante prevalentes em indivíduos quedas foram mais frequentes no período matutino, 10 (31,2\%), ocorrendo em maior frequência, no momento de transferência entre o banheiro e a cama. Quanto aos fatores que possam ter contribuído para queda, identificados a partir dos registros de prontuário, foram: ao levantar-se da cama sem acompanhante e ida ao banheiro (Tabela 3). hospitalizados e, dessa forma, tornam-se um fator importante a ser considerado na predição do risco de queda ${ }^{(9-10,22-24)}$. A faixa etária da população, 
deste estudo, evidencia o predomínio de pacientes idosos, em consonância com dados globais. Vale ressaltar que, indivíduos com mais de 65 anos apresentam declínio funcional, enfraquecimento da saúde, aumento da fragilidade, mais comorbidades, em especial neurológicas, e vulnerabilidade à mortalidade ${ }^{(11,22,25)}$, além do aumento de chance de quedas com o progresso etário ${ }^{(10,15,22,26-27)}$.

Embora a literatura não apresente um consenso sobre o sexo como fator de risco para quedas ${ }^{(28)}$, alguns estudos apontam que há maior incidência de quedas no sexo feminino ${ }^{(20,28-30)}$. Isso pode ser justificado pela diminuição de força de massa muscular, maior contato com as atividades domésticas, alterações hormonais como a redução do estrógeno, com consequente perda de massa óssea e presença de osteoporose ${ }^{(20,28-30)}$. Em contrapartida, estudos citam que a ocorrência de quedas em indivíduos do sexo masculino possa ser explicada, em partes, por fatores culturais, como o fato de não aceitar auxílio para determinadas tarefas como, por exemplo, levantar da cama e deambular ${ }^{(15,20,28,31-32)}$. No presente estudo, a frequência de quedas foi semelhante em ambos os sexos.

Os pacientes, deste estudo, apresentaram risco elevado para queda, em todos os períodos avaliados: no dia da queda, no dia que antecedeu e sucedeu o evento, portanto com pontuações médias inferiores a demonstradas em outros estudos $^{(17,28)}$. Um estudo realizado com pacientes clínicos cirúrgicos com idade média de 58,1 anos (DP 15,4), demonstrou um MFS médio de 31,7 pontos, indicando risco moderado de quedas ${ }^{(17)}$. Outro estudo brasileiro, cujo objetivo foi analisar os fatores de risco para quedas, nas primeiras 48 horas de hospitalização em unidades clínicas e cirúrgicas, com indivíduos com mediana de idade de 54 anos, encontrou-se uma pontuação média da MFS de 39,1, indicativo de risco moderado para quedas (28). Estudos internacionais demonstram valores médios de MFS mais elevados ${ }^{(18)}$. Esses achados distintos $^{(17-18,28)}$ podem ser resultado da diversidade de características clínicas (idade, comorbidades, grau de dependência para a realização de atividades de vida diária e deambulação, estado mental) apresentadas pelos pacientes de cada estudo.

Estudos relatam o aumento médio do tempo de permanência hospitalar em 12,3 dias em função do tratamento dos danos resultantes do incidente de queda, bem como aumento nos custos hospitalares ${ }^{(15,20,26,30)}$. O tempo médio de internação, na amostra estudada, foi de 18.6 dias, entretanto, neste estudo, não foi avaliado o incremento do tempo de internação, em decorrência do episódio de queda.

Das quedas avaliadas, no presente estudo, $8(25 \%)$ resultaram em algum tipo de dano, destes prevaleceram os danos leves $5(15,6 \%)$, seguidos por danos moderados em 3 pacientes $(9,4 \%)$ e nenhum dano grave. Com relação ao grau de severidade do dano, verificou-se que as quedas com danos leves foram as mais prevalentes, semelhantes a outros estudos reportados, na literatura, onde as escoriações/abrasões e contusões foram as lesões mais frequentes ${ }^{(27,31)}$.

Acerca do período do dia de ocorrência da queda, os dados deste estudo são divergentes de outras pesquisas, nas quais a maior ocorrência de quedas ocorreu no turno da noite (27-28). Essa divergência poderia ser explicada por uma possível subnotificação de quedas nesse período. Acredita-se que, durante a noite, muitos pacientes não solicitam auxílio para realizar as atividades, por superestimar suas capacidades físicas ou por constrangimento ${ }^{(15,28)}$. No turno da manhã, a ocorrência de quedas está relacionada às atividades de higiene, curativos, deslocamentos para realizações de exames e eliminações fisiológicas ${ }^{(20,27)}$.

O comprometimento neurológico é um fator de risco conhecido para o maior risco de quedas. Em um estudo caso-controle, realizado com 358 pacientes, em 12 unidades clínicas e cirúrgicas de um hospital universitário da região sul do Brasil, a desorientação e a agitação foram reportadas como fatores associados ao risco de quedas (OR 4,45 $\mathrm{p}<0,001)^{(20)}$. No presente estudo, $34,4 \%$ dos pacientes apresentaram delirium ou comprometimento neurológico.

A terapia medicamentosa é um aspecto importante a ser considerado, uma vez que estes podem potencializar os riscos para a ocorrência de quedas, por promover efeitos adversos como desequilíbrio postural, fraqueza muscular, hipotensão, confusão mental e delirium ${ }^{(11,20,25,30)}$. Dentre os medicamentos potencializadores para o risco de quedas, pode-se citar os medicamentos que atuam no sistema nervoso central e aqueles que atuam no sistema cardiovascular como: hipnóticos, antipsicóticos, ansiolíticos, benzodiazepínicos, opioides, barbitúricos, antihistamínico, anticonvulsivantes, sedativos, antidepressivos, laxantes, anti-hipertensivos e diuréticos ${ }^{(9,20,25)}$. 
Em pacientes cardiológicos é frequente o uso de medicamentos que potencializam o risco de quedas, os quais, em geral, podem afetar o débito cardíaco e a perfusão cerebral(20). Uma avaliação de 86 pacientes com diagnóstico médico de isquemia cardíaca, de um hospital especializado de Fortaleza/Ceará/Brasil evidenciou que $98,8 \%$ das pacientes vítimas de quedas faziam o uso de anti-hipertensivos, diuréticos e narcóticos ${ }^{(25)}$. No presente estudo, 31 $(96,9 \%)$ pacientes faziam uso de medicamentos para doenças crônicas, especialmente doenças cardiovasculares.

Em relação às medidas físicas e estruturais preventivas, recomenda-se a implementação, no ambiente hospitalar, de medidas de segurança, como grades, pisos antiderrapantes, barras de apoio, realização da evolução do estado do paciente, a cada dia, e observação contínua $(10,22)$. Além disso, é importante dissipar a cultura de segurança do paciente no hospital e/ou ambiente de saúde e conscientizar a equipe da importância da notificação dos eventos, em especial entre a equipe de enfermagem. Nesse contexto, é de extrema relevância a atenção da equipe de enfermagem no sentido de prevenir a ocorrência de quedas, estratificando o risco e implementando uma assistência qualificada, de acordo com a demanda de cuidados, sendo necessário considerar fatores intrínsecos e extrínsecos ao paciente ${ }^{(10-11,17,20,29)}$.

É importante salientar que, no ano de 2016, foram implementadas medidas preventivas para a ocorrência de quedas na unidade pesquisada, como adequação de estrutura física, capacitação da equipe, conscientização dos pacientes e familiares, o que resultou em um decréscimo importante da taxa de quedas, nesse ano $(1,5$ quedas/1.000 paciente-dia em 2016), comparadas ao ano anterior (2,7 quedas/1.000 paciente-dia em 2015).

Os dados, deste estudo, contribuem para o conhecimento da temática, sobretudo diante da constatação de que o perfil epidemiológico e demográfico de cada população deve ser conhecido, além de subsidiar a proposição de novos estudos que visem a explorar questões relacionadas aos fatores preditores de queda, visando a estratégias implementadas pelos enfermeiros que são primordiais, no contexto de segurança do paciente, para proporcionar uma assistência qualificada.

Salienta-se que, para a implementação e execução efetiva dos protocolos na prática clínica, é importante o envolvimento da equipe multiprofissional, o entendimento da demanda dos pacientes, durante o período de internação e, dessa forma, o gerenciamento hospitalar deve possibilitar a análise, revisão e a proposição de soluções para novos problemas, além da avaliação constante das intervenções educativas e preventivas.

\section{CONCLUSÃO E IMPLICAÇÕES CLÍNICAS}

No período de estudo, houve um decréscimo de mais de $50 \%$ na taxa de quedas, na unidade de internação cardiológica estudada, 2,7 quedas/1000 pacientes dia em 2015 para 1,5 no ano seguinte. Houve predomínio do evento de queda em pacientes maiores de 60 anos, admitidos, predominantemente, por desordens clínicas, independente do sexo.

Os pacientes do estudo, apresentaram estratificação de risco elevada para quedas, em todos os momentos avaliados, em $25 \%$ das quedas houve algum tipo de dano, com maior frequência de danos leves. Observou-se a necessidade de maior cuidado com os idosos, durante o período matutino, pois as quedas foram mais frequentes, neste período, ocorrendo em maior frequência com atividades relacionadas a idas ao banheiro e saída da cama.

Ressaltamos como limitação do estudo, o tamanho da amostra, o uso de dados secundários de prontuários eletrônicos ou registro de notificação do evento adverso da instituição, bem como a análise de somente uma unidade de internação. Entretanto, as contribuições fornecidas pelos registros de eventos adversos, deste estudo, facilitaram a identificação dos fatores de risco, demonstrando a necessidade de se propor intervenções de enfermagem preventivas, uma vez que assumir o evento e identificar suas causas são maneiras de praticar uma assistência de enfermagem segura ao paciente.

\section{REFERÊNCIAS}

1- World Health Organization (WHO). Conceptual framework for the International Classification for Patient Safety: final technical report. Genebra: WHO; 2009.

2- Oliveira RM, Leitão IMTA, Silva LMS, Figueiredo SV, Sampaio RL, Gondim MM. Estratégias para promover segurança do paciente: De identificação dos riscos às práticas baseadas em evidências. Esc 
Anna Nery 2014;18(1): 122-9. DOI: $10.5935 / 1414-$ 8145.20140018

3- Capucho HC, Arnas ER, Cassiane SHB. Segurança do paciente: Comparação entre notificações voluntárias manuscritas e informatizadas sobre incidentes em saúde. Rev Gaúcha Enferm 2013;34(1): 164-72. DOI: 10.1590/S1983-14472013000100021

4- Agência Nacional de Vigilância Sanitária. Assistência segura: Uma reflexão teórica aplicada à prática. Brasília: ANVISA; 2013.

5- Agência Nacional de Vigilância Sanitária. Incidentes Relacionados à Assistência à Saúde 2015. Boletim Segurança do Paciente e Qualidade em Serviços de Saúde 2016 [citado em 18 ago 2020]. Acesso em: https://www20.anvisa.gov.br/segurancadopacien te/index.php/publicacoes/category/boletins-

estatisticos

6- Silva CF, Reiniack S, Souza BM, Cun KCS. Prevalência dos fatores de risco intrínsecos ao paciente e o desfecho queda na clínica cirúrgica. Cogitare Enferm. 2016 [acesso em 2017 nov 12]; 21(5): 01-08. Disponível em: http://revistas.ufpr.br/cogitare/article/view/4534 $\underline{2 / p d f}$

7- Yunchuan ZPR, Marjorie BPR, Jianghua HP, Heejung KPR, Shin HPPR, Nacy DPR. Evidence on Fall and Injurious Fall Prevention Interventions in Acute Care Hospitals. JONA: The Journal of Nursing Administration [internet]; 2019 [citado em 20 jan 2020]; 49(2):86-92. Acesso em: 10.1097/NNA.0000000000000715

8- Luzia MF, Cassola TP, Suzuki ML, Dias VLM, Pinho LB, Lucena AF. Incidência de quedas e ações preventivas em um Hospital Universitário. Rev Esc Enferm USP 2018;52:1-7. DOI: 10.1590/s1980220x2017024203308

9- Silva AKM, Costa DCM, Reis AMM. Fatores de risco associados as quedas intra-hospitalares notificadas ao núcleo de segurança do paciente de um hospital de ensino. Einstein 2019;17:(1):eAO4432. DOI:

\subsection{4/einstein journal/2019ao4432}

10- Lee S, Lee C, Rodiek S. Neighborhood factors and fall-related injuries among older adults seen by emergency medical service providers. Int J Environ Res Public Health 2017;14(2):E163. DOI: 10.3390/ijerph14020163

11- Herreraa DH, Elizarraraz NA, Argote MV, Quirante NG Hildalgo HC, Hernánde LI. Aplicación de las actividades de la intervención de enfermería Prevención de caídas en adultos hospitalizados.
Enferm Univ. 2017;14(2):118-23 DOU:

10.1016/j.reu.2017.02.007

12- Bittencourt VLL, Graube SL, Stumm EMF, Battisti IDE, Loro MM. Factors associated with the risk of falls in hospitalized adult patients. Rev Esc Enferm USP 2017;51(03):237. DOI: 10.1590/s1980-220x2016037403237

13- Cedraz RO, Gallach $\mathrm{CH}$, Pérez Junior EF, Gomes HF, Rocha RG, Mininel VA. Gerenciamento de riscos em ambiente hospitalar: Incidência e fatores de riscos associados à queda e lesão por pressão em unidade clínica. Esc Anna Nery 2018;22(1):e20170252. DOI: 10.1590/2177-9465ean-2017-0252

14- Berkova H, Berka Z. Falls: A significant cause of morbidity and mortality in elderly people. Vnitr Lek. 2018 [citado em 24 jan 2019]; 64(11):107683. Acesso em: https://www.unboundmedicine.com/medline/cit ation/30606025/Falls: a significant cause of $\mathrm{m}$ orbidity and mortality in elderly people

15- Meneguin S, Ayres JA, Bueno GH. Caracterização das quedas de pacientes em hospital especializado. Rev Enferm UFSM 2014;4(4):784-91. DOI: 10.5902/2179769213554 16- Muray $\mathrm{M}$, Bélanger $\mathrm{CH}$, Razmak J. Fall prevention strategy in an emergency department. Int J Health Care Qual Assur. 2018;31(1):2-9. DOI: 10.1108 / IJHCQA-09-2016-0122

17- Urbanetto JS, Pasa TS, Bittencout HR, Franz F, Rosa VPP, Magnago TSBS. Análise da capacidade de predição de risco e validade da Morse Fall Scale versão brasileira. Rev Gaúcha Enferm. 2017;37(4): e62200. DOI: $10.1590 / 1983-1447.2016 .04 .62200$ 18- Costa-Dias M, Ferreira $P$, Oliveira $A$. Adaptação cultural e linguística e validação da Escala de Quedas de Morse. Rev Enferm Referen. 2014;4(2):7-17. DOI: 10.12707/riii1382

19- Urbanetto JS, Creutzberg M, Franz F, Ojeda BS, Gustavo AS, Bittencourt HR, et al. Morse Fall Scale: Tradução e adaptação transcultural para a língua portuguesa. Rev Esc Enferm USP 2013;47(3):569-75. DOI: 10.1590/50080$\underline{623420130000300007}$

20- Severo IM, Kunchenbecker RS, Vieira DFV, Lucena AF, Almeida MA. Fatores de risco para quedas em pacientes adultos hospitalizados: Um estudo caso-controle. Rev Latino-Am Enfermagem 2018;26:1-9. DOI: 10.1590/1518-8345.2460.3016 21- STrengthening the Reporting of OBservational Studies in Epidemiology. Checklist of items that should be included in reports of cross-sectional studies [cited 2020 July 20]. Available in: https://strobe- 
statement.org/fileadmin/Strobe/uploads/checklis ts/STROBE checklist v4 cross-sectional.pdf 22- Bell SP, Vasileyskis EE, Saraf AA, Jacobsen $J M L$, Kripalani S, Mixon AS, et al. Geriatric syndromes in hospitalized older adults discharged to skilled nursing facilities. J Am Geriatr Soc. 2016;64(4):715-22. DOI: 10.1111/igs.14035

23- Duarte GP, Santos JLF, Lebrãoll ML, Duart YAO. Relação de quedas em idosos e os componentes de fragilidade. Rev Bras Epidemiol. 2018;21(Suppl 2):E180017. DOI: 10.1590/1980549720180017.supl.2

24- Gazibara T, Kurtagic I, Kisic-Tepavcevic D, Nurkovic S, Kovacevic N, Gazibara T, et al. Falls, risk factors and fear of falling among persons older than 65 years of age. Psychogeriatrics 2017;17(4):215-23. DOI: 10.1111/psyg.12217

25- Kruschke C, Butcher HK. Evidence-based practice guideline: Fall prevention for older adults. J Gerontol Nurs. 2017;43(11): 15-21. DOI: 10.3928/00989134-20171016-01

26- Callis N. Falls prevention: Identification of predictive fall risk factors. Appl Nurs Res. 2016;29:53-8. DOI: 10.1016/j.apnr.2015.05.007

27- Rosa VPP, Cappellari FCBD, Urbanetto JS. Análise dos fatores de risco para queda em idosos institucionalizados. Rev Bras Geriatr Gerontol. 2019; 22(1):1-13. DOI: 10.1590/198122562019022.180138

28- Mata LRF, Azevedo C, Policarpo AG, Moraes JT. Fatores associados ao risco de queda em adultos no pós-operatório: Estudo transversal. Ver Latino-Am Enfermagem 2017;22(2):262-8. DOI: 10.1590/1518-8345.1775.2904

29- Gomes GAO, Cintra FA, Batista FS, Neri AL, Guariento ME, Sousa MLR, et al. Elderly outpatient profile and predictors of falls. Sao Paulo Med J. 2013;131(1):13-8. DOI: $\quad$ 10.1590/S1516$\underline{31802013000100003}$

30- Sakai AM, Rossaneis MA, Haddad MCFL, Vituri DW. Risco de queda do leito de pacientes adultos e medidas de prevenção. Rev Enferm UFPE 2016;10(6):4720-6. DOI: 10.5205/reuol.820071830-3-SM.1006sup201602

31- Luzia MF, Prates CG, Adorna JB, Moura GMS. Características das quedas com dano em pacientes hospitalizados. Rev Gaúcha Enferm. 2019; 40(nesp): e20180307. DOI: 10.1590/1983$\underline{1447.2019 .20180307}$

Nota: Pesquisa oriunda de trabalho de conclusão de curso. Agradecimentos ao Hospital do Coração do Brasil, Brasília, Brasil, como local de pesquisa.
Recebido em: 21/09/2020

Aprovado em: $14 / 12 / 2020$

Endereço de correspondência:

Priscilla Roberta Silva Rocha.

Campus Universitário, s/n, Centro Metropolitano, Brasília DF, 72220-275

Email: priscillarocha@unb.br 